\title{
Integración del internet de las cosas en los procesos logísticos de máquinas dispensadoras
}

\section{Integration of internet of things in logistic processes of vending machines}

\author{
Dyron Ramírez \\ Docente, Politécnico Colombiano Jaime Isaza Cadavid, Medellín, Colombia.dyronramirez@elpoli.edu.co
}

Recibido: junio 04, 2018. Aceptado: Julio 15, 2018.

\begin{abstract}
Resumen
Este artículo tiene como principal objetivo definir las condiciones en las que se desarrollan algunos procesos logísticos de las organizaciones en la integración de dispositivos en objetos cotidianos conectados a internet y que, por este medio, entregan información generadora de dinámicas diferentes a las tradicionales en cada una de las actividades que conforman la labor de abastecimiento, para garantizar la disponibilidad del producto en las condiciones que el cliente lo desea y, en este caso, en las actividades enmarcadas en el denominado vending: comercialización de productos como snacks, bebidas, artículos de aseo, medicamentos básicos, entre otros, a través de dispensadores electrónicos. La metodología usada está basada en el análisis de la bibliografía especializada en el tema del vending, el Internet de las Cosas y de las "cosas" que demandan constante nivel de abastecimiento de productos dadas las características de suministro permanente a los clientes por parte de estas, como los dispensadores de snacks, refrescos y de productos perecederos tales como frutas, productos frescos y productos lácteos, así como la consulta en fuentes primarias entre las que se encuentran mediciones sobre comportamiento de agotados y frecuencia de llegada de clientes en tres máquinas ubicadas en una entidad educativa. Igualmente, se evidencia la importancia de utilizar la tecnología para la recolección de la información pertinente, ya sea con la interacción de tecnologías blandas (software) y tecnologías duras o la utilización exclusiva de software, decisión en la que juega un papel preponderante el costo del producto.
\end{abstract}

Palabras Clave: abastecimiento, distribución, Internet de las Cosas (loT), logística, transporte, vending.

\begin{abstract}
The primary objective of this article is the review of the conditions for the application of the Internet of Things in some of the logistic processes. This integration is changing the way for delivering information to the operators, and it is generating different dynamics inside the traditional activities that make up the supply process. Usually, the operators must guarantee the availability of the product in the conditions that the client wishes. For this particular case, the possible applications of the Internet of Things are framed in the so-called "VENDING" business: the marketing of products such as snacks, beverages, toiletries, essential medicines, among others, through electronic dispensers. The methodology used is based on the analysis of the specialized literature on the subject of vending, the Internet of Things and the "things" that demand a constant level of product supply. Also, this work describes the characteristics of the permanent supply in dispensers of snacks, soft drinks and perishable products such as fruits, fresh products, and dairy products. In this regard, the consultation of primary sources in charge of the operation of three vending machines located inside an educational institution was significant. This exploration highlighted aspects such as the measurements on exhausting behavior and frequency of arrival of clients. These factors are identified and discussed for enhancement through the application of the Internet of Things technologies in vending machines.
\end{abstract}

Keywords: Supply, distribution, Internet of things (IoT), Logistics, transport, vending

\section{INTRODUCCIÓN}

El abastecimiento eficiente de productos es una de las actividades más complejas dentro de los procesos logísticos, entendiendo el abastecimiento como la administración de los inventarios y la gestión del nivel de existencias. Al respecto, Vidal expresa [1]: "Siempre tenemos demasiado de lo que no se vende o se consume y muchos agotados de lo que sí se vende o se consume"; sin embargo, esta dinámica ha cambiado pues la política de "cero inventarios" prima en las organizaciones.

Para mejorar los niveles de desempeño de cualquier proceso se busca introducir innovaciones a través de diferentes estrategias y herramientas tecnológicas [2]. Entre ellas, se aplican tecnologías del Internet de las Cosas (loT - Internet of Things), que no es más que la integración de la tecnología de conexión de cualquier

\footnotetext{
Citar como:

D. Ramírez. "Integración del internet de las cosas en los procesos logísticos de máquinas dispensadoras". Revista CINTEX, Vol. 23(1), pp. 25-30. 2018.
} 
Integración del internet de las cosas en los procesos logísticos de máquinas dispensadoras Integration of internet of things in logistic processes of vending machines

objeto que requiera intercambiar información a través de la red [3]. En este escrito se propone evidenciar cómo mitigar el efecto que tiene el abastecimiento deficiente en las máquinas tipo vending a través de la integración con tecnologías propias del loT y la gestión correcta de la información.

El documento se estructuró a partir de la definición de conceptos como gestión de la cadena de suministro, IoT, abastecimiento y vending. Se realizó una muestra puntual en la que se analizó presencialmente el comportamiento de tres máquinas ubicadas en sitios públicos y de gran afluencia y a las que se tenía acceso a información por la integración de quien las administra y elabora la solicitud de abastecimiento. Estas máquinas, en específico, comercializan snacks; igualmente se realizó el análisis bibliográfico para las reflexiones pertinentes; finalmente, se entregarán las conclusiones y las referencias utilizadas.

\section{METODOLOGÍA PARA LA REVISIÓN DE LITERATURA}

El presente documento sigue una línea que comprende desde una visión general de la cadena de abastecimiento, las máquinas vending y el abastecimiento, pasando por el significado del Internet de las Cosas hasta conceptos que implican la utilización de esta estrategia. Para comenzar este trabajo se realizó una búsqueda bibliográfica de la información desde las diferentes fuentes, entre ellas, artículos de bases de datos académicas, libros, informes, periódicos, comunicaciones científicas, revistas, tesis de grado y otras publicaciones seriadas, además de páginas web especializadas.

Las bases de datos académicas consultadas fueron:

- Google Scholar,

- Science Direct,

- IEEE Xplore.

Los términos de búsqueda (keywords) que se seleccionaron fueron los siguientes:

- Internet of Things AND Logistics,

- Internet of Things AND Supply Chain,

- Internet of Things and vending,

- IoT and Logistics,

- IoT and Supply Chain,

- IoT and vending,

- IoT and replenishment,

- Vending machine replenishment,

- Vending machine supply chains,

- Vending machine controller.

Se procuró obtener información de escritos e investigaciones más actualizados mediante la búsqueda de las últimas tendencias y, sobre todo, con evidencias de la aplicabilidad del loT en el tema específico, aunque existen conceptos que no pierden vigencia y fueron tomados de fuentes menos contemporáneas. Es necesario aclarar que se ha investigado mucho sobre este tema, incluso existen otras aplicaciones vinculadas al loT, como el pago con dinero plástico, y que en la medida en que la tecnología se incrusta físicamente en la máquina, las aplicaciones que podrán integrarse a ellas serán muchas más.

\subsection{Definiciones de los elementos básicos}

La definición de cadena de suministro es expresada como un conjunto de tres o más empresas conectadas o relacionadas con flujos de productos, servicios, finanzas e información desde el suministro hasta el cliente final [4].

El Internet de las cosas (IoT - Internet of Things) es un paradigma donde los objetos cotidianos pueden equiparse con capacidades de identificación, detección, conexión en red y procesamiento que les permitan comunicarse entre sí y con otros dispositivos y servicios a través de Internet para lograr algún objetivo. En última instancia, los dispositivos loT serán ubicuos, conscientes del contexto y habilitarán la inteligencia ambiental [4]. En el contexto de los problemas de logística y abastecimiento, el concepto por sí solo no tiene valor; sin embargo, loT se convierte en una herramienta esencial para las cadenas de suministro que introducen esta tecnología, cuando los dispositivos conectados son capaces de comunicarse entre sí e integrarse con sistemas de inventario gestionados por el proveedor, sistemas de soporte al cliente, aplicaciones de inteligencia empresarial y análisis de negocio [5]

En cuanto al abastecimiento se puede afirmar que es el proceso que se encarga de garantizar el suministro, almacenamiento y distribución en los frentes de trabajo, además de la estimación de las cantidades de recursos por usar [6].

Es necesario, por último, definir el elemento en el que se pretende aplicar la propuesta: las máquinas de vending son equipos expendedores automáticos de todo tipo de artículos y productos comestibles. La acción de 
suministrar dichos artículos mediante estas máquinas se conoce internacionalmente como vending [7]. Las máquinas comercializan desde alimentos como snacks, pasabocas, gaseosas, café, hasta medicinas de libre venta y artículos electrónicos o gadgets como cables USB, memorias extraíbles, cargadores para celular o elementos pequeños de ferretería.

\section{CARACTERÍSTICAS DEL PROCESO DE ABASTECIMIENTO DE MÁQUINAS TIPO VENDING}

Para el caso que convoca es necesario ubicarse específicamente en la operación logística y en la dificultad de la comercialización de productos en dispensadores en el proceso de resurtido y minimización de agotados.

En la gestión del inventario de las máquinas vending, ubicadas en los sitios de los clientes, a menudo se adoptan estrategias de inventario administrado por el vendedor (VMI). Con VMI, los vendedores son responsables de todas las decisiones relacionadas con la administración del inventario del cliente [8]; esta constituye una de las mayores dificultades, pues cuando se incrementa la demanda y el vendedor acaba de surtir la máquina, debe esperar hasta que sea tiempo de regresar por la ruta, es decir, el control de las decisiones de reposición reside en los proveedores en lugar de sus clientes.

Los proveedores, específicamente el vendedor, requieren que sus clientes proporcionen información sobre ventas de productos, niveles actuales de inventario, fechas para la recepción de mercancías y existencias muertas y devoluciones [9]; información que a menudo no se encuentra disponible, dado que las comunicaciones en este negocio aún se dan de manera manual, es decir, no se encuentran en línea.

Es en este punto donde entra a jugar un papel preponderante el Internet de las Cosas. Su importancia está fundamentada en el aporte que le puede dar la conexión a internet de las máquinas dispensadoras de tal manera que cuando se presenten agotados o se supere el stock de seguridad, la información viaje al sistema del VMI para que este se desplace hasta el punto de ubicación de la máquina con el fin de solucionar el escenario que se presenta.

\subsection{Recolección de información de estado de abastecimiento}

La conexión de la máquina a internet y el intercambio de información permite determinar cuándo se superó el stock de seguridad, cuándo se vende una unidad de cada producto y cuándo hay agotados, y, por supuesto, la generación de información que permita el análisis y la retroalimentación pertinente y permanente. Para ello se tienen en cuenta las siguientes consideraciones:

3.1.1 En el momento inicial, la demanda está establecida a partir de históricos con un promedio sobre los consumos.

3.1.2 El surtido se realiza periódicamente, con un promedio de cada 1,4 días (este dato resulta de dividir los 7 días de la semana entre los 5 días hábiles dado que, aunque la máquina está disponible las 24 horas los 7 días a la semana, los puntos donde se ubican regularmente tienen este límite de operación).

3.1.3 Igualmente, las máquinas poseen un inventario de seguridad limitado por el espacio que tiene cada posición asignada al producto y que depende del volumen y la profundidad de la máquina.

3.1.4 Por operatividad, los picos de consumo se generan por la ejecución de eventos especiales en los que aumenta el número de clientes y por supuesto varía el tipo de producto que se consume.

\subsection{Proceso de surtido de las máquinas de vending}

El proceso de surtido de las máquinas ubicadas en una institución de educación superior que se describe a continuación fue extraído de la recolección de información de una fuente primaria vinculada a la labor. Este proceso se repite periodo a periodo:

3.2.1. Las máquinas inician con un inventario inicial que corresponde a variedad y lleno completo de las aproximadamente 60 ubicaciones que conforman una máquina que expende snacks.

3.2.2. Existe un rutero (persona que regularmente visita cada máquina de acuerdo con una ruta que le es asignada), quien completa, conforme la mercancía que posea en su vehículo, los artículos faltantes de forma que la máquina quede con el mayor surtido posible.

3.2.3. Si la máquina tiene un agotado y, si producto de la ruta, el empleado no tiene las cantidades necesarias para remplazarlas, la consecuencia será que el producto estará agotado hasta que la ruta lo lleve de nuevo a ese punto.

3.2.4. Por el contrario, si el empleado lleva una cantidad de productos de acuerdo con la demanda promedio y esta ha tenido un pico hacia la baja, debe regresar entonces con los sobrantes o con la totalidad en el caso extremo.

3.2.5. A los vehículos les es asignado una cantidad de mercancía para atender el número total de máquinas asignadas y que corresponde a la demanda promedio del total de máquinas.

3.2.6. No se tienen en cuenta picos de consumo fruto de eventos o mayor afluencia de clientes 
Integración del internet de las cosas en los procesos logísticos de máquinas dispensadoras Integration of internet of things in logistic processes of vending machines

Estas condiciones llevan a que se presenten dificultades de disponibilidad, especialmente en los momentos en los que se realizan eventos especiales que convocan un número inusitado de potenciales clientes, lo que lleva a un aumento de la demanda para la que no se está preparado y que puede generar faltantes de algunas referencias. Para el logro del objetivo de satisfacer la demanda en esos casos se debe establecer un inventario de seguridad (SuS), dado por la expresión [10]:

$$
\left.S s=u * \sqrt{\left(D m^{2}\right.} \sigma^{2}+D \lambda^{2}\right) \text {, }
$$

Donde $D m$ es la demanda media, $D$ es el tiempo promedio de abastecimiento, (1.4 días), $\sigma$ es la desviación estándar de la demanda y $\lambda$ es la desviación estándar del tiempo de aprovisionamiento.

Otro aspecto a tener en cuenta es el proceso de selección de productos descrito por Hiraishi y Nemoto [11] es el modelo más cercano a lo que se puede observar y que coincide con las siguientes variables de comportamiento del público que utiliza los dispensadores: el atributo del cliente no tiene en cuenta el género (masculino o femenino), pues ), cuando los clientes compran productos que se ofertan en las máquinas expendedoras, seleccionan un artículo para comprar, y el artículo que se comprará cambia de manera probabilística sin tanta importancia del sexo o la ubicación del cliente; el mayor factor de importancia en la decisión de compra del artículo está en la disponibilidad del inventario [11].

\section{DISCUSIÓN DE LAS APLICACIONES DE IOT EN EL ABASTECIMIENTO DE MÀQUINAS TIPO VENDING}

Para el control del inventario existe tecnología de procesamiento de información en tiempo real basada en RFID y NFC que puede realizar un monitoreo en tiempo real de casi todos los eslabones de la cadena de suministro [12], desde el diseño de productos básicos, compras de materias primas, producción, transporte, almacenamiento, distribución y venta de semielaborados, y productos terminados, para nuestro caso, y devuelve 'procesamiento y servicio postventa. También es posible obtener información relacionada con los productos de manera puntual, oportuna y precisa para que las empresas, o incluso toda la cadena de suministro, puedan responder a mercados intrincados y cambiantes en el menor tiempo posible [13].

El impacto de los desarrollos de loT en el entorno industrial se ha estudiado antes desde el punto de vista de los sistemas ciber-físicos y la industria 4.0 [14], con sus principales áreas de utilización siendo revisadas en [3], [5], [13], y en particular, discutiendo la implementación en el apoyo de cadenas de suministro y máquinas expendedoras automatizadas [7], [8], [11]. El principal resultado de la aplicación loT radica en que el tiempo de reacción de las empresas tradicionales es de 120 días, desde los requisitos de los clientes hasta el suministro de productos básicos, mientras que las empresas avanzadas que hacen uso de estas tecnologías (como Wal-Mart y Metro) solo necesitan unos pocos días y básicamente puede funcionar sin existencias de seguridad, escalando este dato a nuestro caso, los tiempos de atención serían unas pocas horas [13].

Sin embargo, en la industria de máquinas expendedoras, el establecimiento de mejor calendario de reabastecimiento es un componente clave para encajar con un control de frecuencia y cantidad. Por lo tanto, es importante para la industria revisar la política de reposición y el sistema de las máquinas expendedoras. El proceso de reposición tradicional se basa en la estimación de los registros de inventario históricos. El problema principal del enfoque existente es utilizar una política de revisión periódica para reponer los productos de las máquinas expendedoras. Esta política solo es adecuada cuando la demanda es estable y el costo de reabastecimiento es muy elevado [15]. La demanda de los productos de la máquina expendedora es fluctuante y el costo de la reposición es bastante alto. Los problemas del enfoque existente causado por la política de revisión periódica son (i) el agotamiento se produce con frecuencia, (ii) control de inventario no confiable, (iii) política de reabastecimiento poco fiable y (v) eficacia de bajo costo [16], [17]. Por lo tanto, la información de inventario precisa e instantánea es otro tema importante para permitir la confiabilidad de la disponibilidad del producto. El proveedor puede usar la información realista para anticipar mejor la demanda futura. Por ello, es fundamental para las empresas de servicios de venta construir una red de información en tiempo real para una mejor gestión de la cadena de suministro con una mejor estrategia de reabastecimiento [18], [19].

Para el caso de los dispensadores de snacks y de productos perecederos, tales como frutas, productos frescos y productos lácteos que son partes vitales de nuestra nutrición, desde la producción hasta los sitios de consumo se cubren miles de kilómetros o incluso más y durante el transporte se debe monitorear el estado de conservación (temperatura, humedad, impacto), igualmente en el almacenamiento y la exhibición para evitar la incertidumbre en los niveles de calidad y de disponibilidad para las decisiones de distribución. En este caso, el nivel de rotación y de monitoreo es más estricto, pues las fechas de vencimiento son más cortas y la cadena de abastecimiento debe estar más sincronizada. Las tecnologías de computación y sensores generalizadas ofrecen un gran potencial para mejorar la eficiencia de la cadena de suministro de alimentos, en este caso, loT es una de ellas [13]. 
Este tipo de aplicaciones son las que se propone implementar en este caso para las máquinas vending, pues, debido al comportamiento de rotación de los productos se pueden presentar casos de desabastecimiento, por ejemplo, por la realización de eventos que aumenten la cantidad de público circulante y, por supuesto, el nivel de consumo. Para evitar agotamientos imprevistos, la utilización de hardware y software a través de soluciones como la integración de medición de consumo a través del conteo de vueltas que pueda dar el serpentín donde se encuentra el producto disponible en su posición o a través de la utilización de etiquetas de identificación de radiofrecuencia (RFID) para cada producto, y la posterior comunicación del estado de inventario de la máquina a través del internet se convierte en un mecanismo tecnológico de gran utilidad en el sector.

Como beneficios de la implementación de la tecnología loT, de acuerdo con Pérez y Barrera [20], quienes citan a Antonio Alandi, se pueden mencionar:

- Ahorro en costo: mejora en los procesos de productividad.

- Mejor utilización de los recursos: hay visibilidad en tiempo real y la cadena de abastecimiento mejora su rendimiento.

- Mejora la productividad: los empleados asimilan conceptos como el justo a tiempo y el aprendizaje en los puestos de trabajo, de esta forma se aprenden tareas de manera mucho más rápida.

\section{CONCLUSIONES}

A lo largo del escrito se describió la situación problemática que se presenta a las máquinas vending en torno al abastecimiento, los niveles de inventario, la confiabilidad del histórico sobre el que se basan para reaprovisionar y, sobre todo, las pérdidas por ventas no realizadas, en especial por la fluctuación de la demanda. Se evidenció la importancia de utilizar la tecnología para la recolección de la información pertinente, ya sea con la interacción de tecnologías blandas (software) y tecnologías duras o la utilización exclusiva de software, decisión en la que juega un papel preponderante el costo del producto.

Además en el artículo se discutió la introducción de otro elemento tecnológico al modelo de negocio: el Internet de las Cosas, a través de la conexión a internet de la máquina luego de la adecuación física que se requiere para que la medición de la salida de productos se interconecte con el sistema de información que posee la máquina y pueda enviarla vía web y, de esta de forma, se tomen las decisiones pertinentes sobre los parámetros principales del suministro de la máquina, tales como número y tipos de unidades vendidas, nivel de inventario de seguridad $y$, por supuesto, planeación del abastecimiento a través de la ruta correcta y con las cantidades y referencias precisas y necesarias.

\section{REFERENCIAS}

[1] C. J. Vidal Holguín, Fundamentos de control y gestión de inventarios. Programa Editorial Universidad del Valle, 2010.

[2] J. J. Castro Maldonado, J. A. Patiño Murillo, and C. Gómez López, "Procesos de I+D+i en el Centro de Servicios y Gestión Empresarial del Servicio Nacional de Aprendizaje SENA 2015-2017,” Rev. Espac., vol. 39, no. 20, p. 21, 2018.

[3] A. Whitmore, A. Agarwal, and L. Da Xu, "The Internet of Things-A survey of topics and trends," Inf. Syst. Front., vol. 17, no. 2, pp. 261-274, Apr. 2015.

[4] J. T. Mentzer, Fundamentals of supply chain management: twelve drivers of competitive advantage. Thousand Oaks, Calif.: Sage Publ, 2004.

[5] I. Lee and K. Lee, "The Internet of Things (IoT): Applications, investments, and challenges for enterprises," Bus. Horiz., vol. 58, no. 4, pp. 431-440, jul. 2015.

[6] J. Echavarría, "Condiciones Laborales y Productivas en Microempresas de Confección de la ciudad de Medellín," Rev. CINTEX, vol. 20, no. 2, pp. 79-95, 2015.

[7] S. Vicini, A. Sanna, and S. Bellini, "A Living Lab for Internet of Things Vending Machines," in The Impact of Virtual, Remote, and Real Logistics Labs, Berlin, Heidelberg, 2012, pp. 35-43.

[8] L.-T. Chen and C.-C. Wei, "Multi-period channel coordination in vendor-managed inventory for deteriorating goods," Int. J. Prod. Res., vol. 50, no. 16, pp. 4396-4413, Aug. 2012.

[9] D. Choudhary, R. Shankar, P. K. Dey, H. Chaudhary, and L. S. Thakur, "Benefits of retailer-supplier partnership initiatives under time-varying demand: a comparative analytical study," Int. J. Prod. Res., vol. 52, no. 14, pp. 4279-4298, Jul. 2014.

[10] R. H. Ballou, Business logistics/supply chain management: planning, organizing, and controlling the supply chain, 5. ed. Upper Saddle River, NJ: Pearson Prentice Hall, 2004. 
[11] G. Nemoto and K. Hiraishi, "Modeling and optimization of item changes in vending machines," in 2017 11th Asian Control Conference (ASCC), 2017, pp. 19-24.

[12] J. A. Patiño, J. J. Espinosa, and R. E. Correa, "A comparison of Kalman-based schemes for localization and tracking in sensor systems," in Communications (LATINCOM), 2010 IEEE Latin-American Conference on, 2010, pp. 1-5.

[13] L. Atzori, A. lera, and G. Morabito, "The Internet of Things: A survey," Comput. Netw., vol. 54, no. 15, pp. 2787-2805, Oct. 2010.

[14] N. Jazdi, "Cyber physical systems in the context of Industry 4.0," in 2014 IEEE International Conference on Automation, Quality and Testing, Robotics, Cluj-Napoca, Romania, 2014, pp. 1-4.

[15] W. See, "Wireless technologies for logistic distribution process," J. Manuf. Technol. Manag., vol. 18, no. 7, pp. 876-888, Sep. 2007.

[16] S.-P. Wang, "On inventory replenishment with non-linear increasing demand," Comput. Oper. Res., vol. 29, no. 13, pp. 1819-1825, nov. 2002.

[17] S. Baptista, R. C. Oliveira, and E. Zúquete, "A period vehicle routing case study," Eur. J. Oper. Res., vol. 139, no. 2, pp. 220-229, Jun. 2002.

[18] S.-P. Wang, "An inventory replenishment policy for deteriorating items with shortages and partial backlogging," Comput. Oper. Res., vol. 29, no. 14, pp. 2043-2051, Dec. 2002.

[19] J. C. Morales Saldarriaga, K. Fernández Morales, and J. E. Pulido, "Evaluación de técnicas de producción accesible en cursos masivos, abiertos y en línea - MOOC," Rev. CINTEX, vol. 21, no. 1, pp.

89-112, 2016.

[20] J. C. Pérez Yepes and S. Barrera Jaramillo, "Juan D. Hoyos Distribuciones: el impacto del internet de las cosas en la logística," Mercatec, no. 53, pp. 49-70, 2018. 